\title{
Optical imaging of L723: the structure of $\mathrm{HH} 223^{\star}$
}

\author{
R. López ${ }^{1}$, R. Estalella ${ }^{1}$, G. Gómez² , and A. Riera ${ }^{3,1}$ \\ 1 Departament d'Astronomia i Meteorologia, Universitat de Barcelona, Av. Diagonal 647, 08028 Barcelona, Spain \\ e-mail: [rosario;robert.estalella]@am.ub.es \\ 2 Instituto de Astrofísica de Canarias, 38200 La Laguna, Tenerife, Spain \\ e-mail: ggv@ll.iac.es \\ 3 Departament de Física i Enginyeria Nuclear, Universitat Politècnica de Catalunya, Av. Víctor Balaguer s/n, \\ 08800 Vilanova i la Geltrú, Spain \\ e-mail: angels.riera@upc.es
}

Received 10 March 2006 / Accepted 23 March 2006

ABSTRACT

\begin{abstract}
Aims. We imaged the Lynds 723 dark nebula (L723) with the aim of studying the morphology of the Herbig-Haro object HH 223 and other line-emission nebula detected in the region.

Methods. We obtained deep narrow-band images in the $\mathrm{H} \alpha$ and [SII] lines and in the continuum nearby $\mathrm{H} \alpha$ of a field of $\sim 5^{\prime}$ of the L723 dark nebula centered on HH 223.

Results. The $\mathrm{H} \alpha$ and [SII] images reveal the detailed morphology of $\mathrm{HH} 223$, unresolved in previous optical images. Both images show a quite complex knotty, wiggling structure embedded in a low-emission nebula. Comparison between the [SII] and $\mathrm{H} \alpha$ fluxes of the knots are indicative of variations in the excitation conditions through $\mathrm{HH} 223$. In addition, several other faint nebula are detected in $\mathrm{H} \alpha$ a few arcmin to the SE and to the NW of $\mathrm{HH} 223$, all of them lying projected onto the east-west pair of lobes of the quadrupolar $\mathrm{CO}$ outflow. Comparison between the $\mathrm{H} \alpha$ and the continuum images confirms the HH-like nature of the Vrba object V83, while the Vrba objects V84 and V85 are identified as faint field stars.
\end{abstract}

Key words. ISM: jets and outflows - ISM: individual objects: L723 - ISM: individual objects: HH 223 - stars: formation ISM: Herbig-Haro objects

\section{Introduction}

Lynds 723 (L723) is an isolate dark cloud located at a distance of $300 \pm 150 \mathrm{pc}$ (Goldsmith et al. 1984) with clear signs of star formation activity. It is one of the few known cases where a quadrupolar $\mathrm{CO}$ outflow has been found. The outflow is formed by two separate pairs of bipolar CO lobes (a larger pair, of $\sim 7^{\prime}$ long, lying in an east-west orientation and another smaller pair, of $\sim 4^{\prime}$ long, lying in a north-south orientation). The four CO lobes emanate from a common center, where the Class 0 source IRAS $19156+1906$ is located. Since the discovery of the outflow, several scenarios have been proposed to explain its peculiar morphology (Goldsmith et al. 1984; Avery et al. 1990; Lee et al. 2002, and references therein).

Anglada et al. (1991) discovered two $3.6 \mathrm{~cm}$ radio continuum sources, VLA1 and VLA2, towards the center of the quadrupolar outflow, both within the error ellipsoide of the IRAS source. Higher resolution observations by Anglada et al. (1996) found that the VLA2 source appears elongated approximately along the axis direction of the east-west $\mathrm{CO}$ bipolar outflow. Their results suggest that this source is a thermal radio jet, being related to the excitation of the east-west $\mathrm{CO}$ outflow. Later observations led to conclude that only VLA2 is a young stellar object (YSO), being associated with the $\mathrm{CO}$ outflow, while VLA1 is most likely a field source. In fact, only VLA2 is associated with circumstellar

* Based on observations made with the $2.6 \mathrm{~m}$ Nordic Optical Telescope operated at the Observatorio del Roque de los Muchachos of the Instituto de Astrofísica de Canarias. gas traced by millimeter continuum emission (Cabrit \& André 1991; Reipurth et al. 1993), and in addition, VLA2 is embedded in high density molecular gas traced by ammonia (Girart et al. 1997) and CS (Hirano et al. 1998).

In spite of the high interest of this region, there are few observations of L723 in the optical and near-infrared ranges. In the near-infrared, there is a $K^{\prime}$ image from Hodapp (1994), where a filamentary nebulosity is visible $\sim 1.5$ northwest of VLA2. A more recent $\mathrm{H}_{2}$ image from Palacios \& Eiroa (1999) shows $\mathrm{H}_{2}$ emission, with several condensations located at both sides of VLA2, and lying projected along the axis direction of the east-west bipolar CO outflow. At optical wavelengths, there are the $\mathrm{R}, \mathrm{I}$ and $\mathrm{H} \alpha$ images of Vrba et al. (1986). The $\mathrm{H} \alpha$ image shows a nearly linear emission structure of $\sim 30^{\prime \prime}$, elongated in the east-west direction and located towards the east of VLA2, at the southern edge of the high extinction region where the radio source is embedded. This structure corresponds to HH 223 in the Reipurth Catalogue of Herbig-Haro (HH) objects (Reipurth 1994). In addition, their optical images show several faint nebulosities that the authors identify as possible $\mathrm{HH}$ objects. All of them, HH 223 and the nebulosities, lie projected onto the lobes of the east-west $\mathrm{CO}$ bipolar outflow. However, these optical images are not deep enough to resolve the HH 223 morphology and they were not acquired in good weather conditions. With the aim of studying with more detail the morphology of the emission in $\mathrm{HH} 223$ and to elucidate whether the optical nebulosities have an $\mathrm{HH}$ nature, as well as to explore the relationship between the optical, molecular and near infrared emissions of L723, 


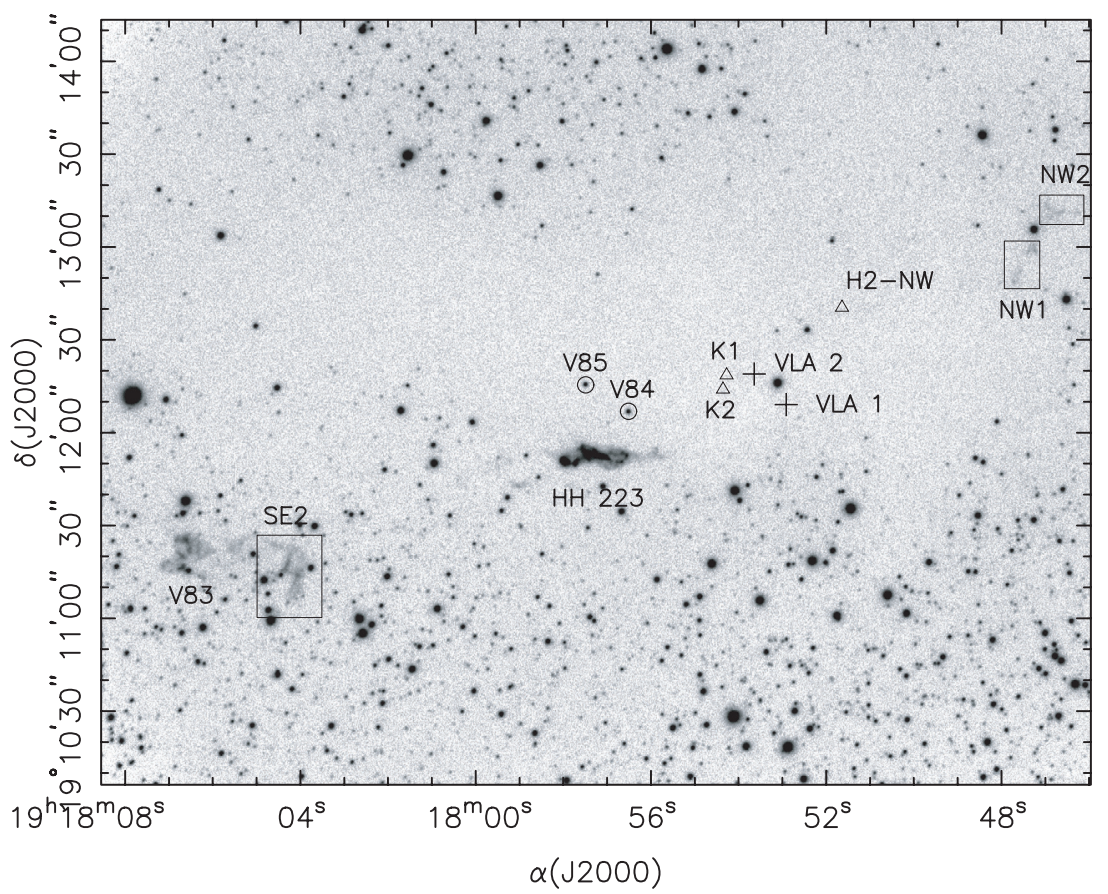

Fig. 1. H $\alpha$ image of the L723 field. HH 223 is at the center of the field. Crosses mark the positions of the VLA sources of Anglada et al. (1996). The positions of the $\mathrm{H}_{2}$ emission peaks K2, K1 and $\mathrm{H}_{2}-\mathrm{NW}$ of Palacios \& Eiroa (1999) have been marked by triangles. Open circles mark the stars that coincide with the positions of the objects V84 and V85 of Vrba et al. (1986). Rectangles mark the new H $\alpha$ nebulosities detected in this work. The nebulosity coinciding with the V83 object of Vrba et al. (1986) (HH 223-SE1 in this work) has also been marked.

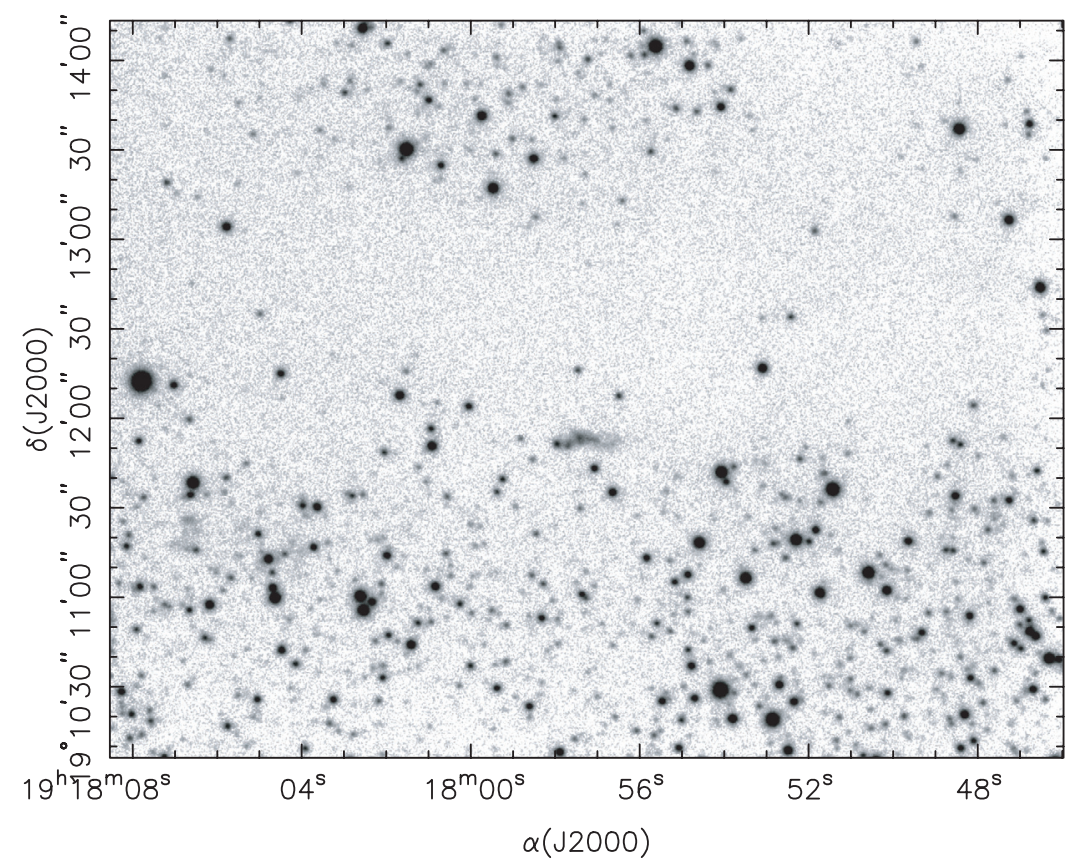

Fig. 2. [SII] image of the L723 field.

we acquired new, deep optical narrow-band images in the $\mathrm{H} \alpha$ and [SII] lines and in the continuum nearby $\mathrm{H} \alpha$ of the L723 field. The results are presented in this paper.

\section{Observations and data reduction}

Deep CCD narrow-band images of L723, which includes HH 223, were obtained with the $2.6 \mathrm{~m}$ Nordic Optical Telescope (NOT) of the Observatorio del Roque de los Muchachos (ORM,
La Palma, Spain) using the Service Time mode facility in two observing runs (July 2004 and September 2005). The images were obtained with the Andalucia Faint Object Spectrograph and Camera (ALFOSC). The image scale was $0{ }^{\prime} 188$ pixel $^{-1}$. The effective imaged field was $\sim 5^{\prime} \times 5^{\prime}$. In July 2004 , an $\mathrm{H} \alpha$ filter (central wavelength $\lambda=6564 \AA$, bandpass $\Delta \lambda=33 \AA$ ) was used to obtain a deep image ( $2.5 \mathrm{~h}$ integration time) in the $\mathrm{H} \alpha$ line by combining five frames of $1800 \mathrm{~s}$ exposure each. In addition, a filter of the continuum nearby $\mathrm{H} \alpha(\lambda=6621 \AA, \Delta \lambda=39 \AA)$ was 


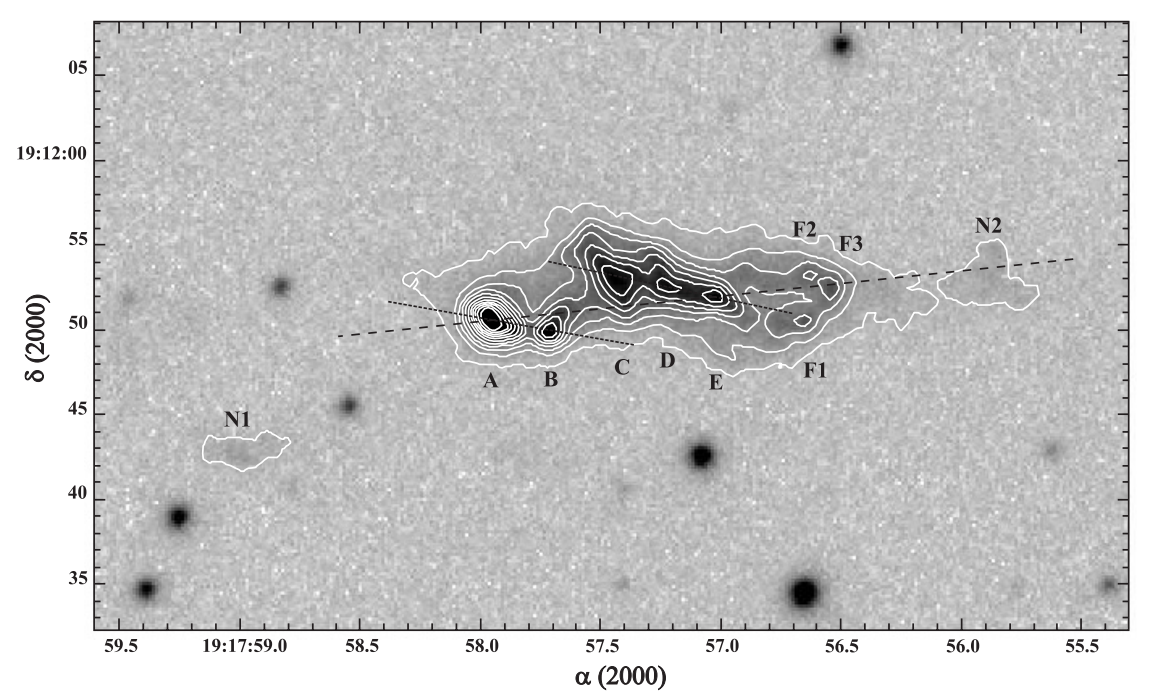

Fig. 3. Close-up of HH 223 and the two adjacent nebulosities N1 and N2 obtained from the H $\alpha$ image of Fig. 1, showing the details of the emission in HH 223. Several knots appear embedded in a low-emission nebula. A contour plot has been superposed to help with the identification of the knots. Dashed and dotted lines mark the orientations refered to in the text.

used to obtain an image of the same field of $900 \mathrm{~s}$ exposure. The range of this continuum filter excludes bright $\mathrm{HH}$ emission lines, being thus useful to discriminate between scattered starlight and pure $\mathrm{H} \alpha$ line emission. Values of the seeing were $0.7-1^{\prime \prime}$. In September 2005, a [SII] filter $(\lambda=6725 \AA, \Delta \lambda=60 \AA)$ that includes the two red [SII] lines, was used to obtain a deep image $(2.5 \mathrm{~h}$ integration time) by combining five frames of $1800 \mathrm{~s}$ exposure each. Values of the seeing were 1 ." $4-1$.' 8 .

All the images were processed with the standard tasks of the $\mathrm{IRAF}^{1}$ reduction package. In order to correct for the misalignments among individual frames, the frames were recentered using the reference positions of fifteen field stars. Astrometric calibration of the three final images was performed in order to compare the positions of $\mathrm{HH} 223$ and other line-emission nebula detected in the field with the positions of the VLA2 radio continuum source, and with the other molecules (i.e., $\mathrm{H}_{2}, \mathrm{CO}$ ). In order to register the images, we used the $(\alpha, \delta)$ coordinates from the USNO-B1.0 Catalogue ${ }^{2}$ of ten field stars well distributed on the observed field. The rms of the transformation was $0 . ' 16$ in both coordinates.

In addition, with the aim of performing a meaningful comparison between the $\mathrm{H} \alpha$ and [SII] emissions of $\mathrm{HH} 223$, we extracted from the [SII] and $\mathrm{H} \alpha$ images of the L723 field two subimages of $85^{\prime \prime} \times 35^{\prime \prime}$ each. Both subimages included HH 223 and ten field stars. The $\mathrm{H} \alpha$ subimage was smoothed with a Gaussian to match the seeing of the [SII] subimage. The background, obtained by averaging the emission of several regions free of stars close to $\mathrm{HH} 223$, was substracted to each image. Finally, the subimages were scaled in flux by applying a scaling factor derived from differential aperture photometry of the common field stars of the two subimages. These two subimages were then used to obtain the $\mathrm{HH} 223$ [SII]/H $\alpha$ flux ratio map that will be discussed later.

1 IRAF is distributed by the National Optical Astronomy Observatories, which are operated by the Association of Universities for Research in Astronomy, Inc., under cooperative agreement with the National Science Foundation.

2 The USNOFS Image and Catalogue Archive is operated by the United States Naval Observatory, Flagstaff Station.

\section{Results and discussion}

Figure 1 shows the $\mathrm{H} \alpha$ line image of L723 and Fig. 2 shows the [SII] image of the same field. At the center of both images there is a bright, extended structure of $\sim 30^{\prime \prime}$ length, elongated approximately along the east-west direction that corresponds to HH 223, first reported by Vrba et al. (1986) as a "linear emission feature". In addition, the image reveals several other fainter nebulosities to the south-east and to the north-west of HH 223. All of these $\mathrm{H} \alpha$ nebulosities are undetected in the image obtained through the narrow-band filter that includes the emission of the continuum nearby $\mathrm{H} \alpha$, indicating thus that all these features are pure emission-line features. We will describe all of them in the following.

\subsection{HH 223 morphology}

\subsection{1. $\mathrm{H} \alpha$ emission}

Our $\mathrm{H} \alpha$ image shows unprecedent details of the morphology of $\mathrm{HH} 223$ and allow us to resolve the structure of the "linear emission feature" reported by Vrba et al. (1986). Our image reveals a quite complex knotty structure, with several condensations embedded in a more diffuse, nebular emission. Figure 3 shows a close-up of the $\mathrm{H} \alpha$ image of $\mathrm{HH} 223$. We have identified several bright knots, labeled from A to F3, from east to west. Table 1 gives the peak positions of the knots. It should be noted that knots A and F2 coincide, respectively, with the positions given by Vrba et al. (1986) for the L723 objects V81 and V82, which they used to mark the eastern and western borders of their "linear emission feature". Furthermore, we detected in the H $\alpha$ image two additional nebular emissions that do not appear in the continuum image. One of these nebulosities (N2) extends over $\sim 11^{\prime \prime}$ to the west of the F3 knot and most probably forms part of the HH 223 object. Another faint, arc-shaped nebulosity (N1) extends over $\sim 3^{\prime \prime} .5$, and is located $\sim 13^{\prime \prime}$ to the SE of HH 223 (see Fig. 3).

Palacios \& Eiroa (1999) detected $\mathrm{H}_{2}$ emission in L723. The emission shows several knots lying along the axis direction of the east-west $\mathrm{CO}$ bipolar outflow. The $\mathrm{H}_{2}$ knots labeled H2-SE by these authors appear to be the near infrared counterpart of $\mathrm{HH}$ 223. A more careful comparison between the near infrared 
Table 1. Peak positions of the $\mathrm{H} \alpha$ knots of $\mathrm{HH} 223$ and $\mathrm{H} \alpha$ nebulosities in $\mathrm{L} 723$.

\begin{tabular}{lccc}
\hline \hline Object & $\alpha_{2000}$ & $\delta_{2000}$ & Notes \\
\hline HH 223 knots: & & & \\
A & 191757.97 & +191150.7 & \\
B & 191757.72 & +191150.1 & \\
C & 191757.43 & +191153.3 & \\
D & 191757.30 & +191153.0 & \\
E & 191757.03 & +191152.1 & \\
F1 & 191756.66 & +191150.9 & $(1)$ \\
F2 & 191756.66 & +191153.4 & $(1)$ \\
F3 & 191756.56 & +191152.9 & $(1)$ \\
H $\alpha$ nebulosities: & & & \\
N1 & 191759.0 & +191142 & $(2)$ \\
N2 & 191755.0 & +191153 & $(2)$ \\
HH 223-SE1 & 191806.1 & +191121 & $(2)$ \\
HH 223-SE2 & 191804.3 & +191116 & $(2)$ \\
HH 223-NW1 & 191747.3 & +191301 & $(2)$ \\
HH 223-NW2 & 191746.8 & +191312 & $(2)$ \\
\hline
\end{tabular}

Notes: (1) Peak positions of the emission enhancements found in the faint extended arc-like filamentary emission westwards of knot E; (2) position of the centroid of the nebulosity.

$\left(\mathrm{H}_{2}\right)$ and the optical $(\mathrm{H} \alpha)$ emissions shows that the H2-SE emission of Palacios \& Eiroa (1999) spatially coincides with the $\mathrm{H} \alpha$ emission from knots $\mathrm{C}$ to $\mathrm{F} 3$. Both emissions also show very similar shapes, although the $\mathrm{H} \alpha$ emission is spatially more extended than the $\mathrm{H}_{2}$ emission. Furthermore a fainter, arc-shaped $\mathrm{H}_{2}$ emission located to the north-west of H2-SE coincides in position with the fainter $\mathrm{H} \alpha$ emission nebulosity N2. Figures 1 and 3 show that the strongest $\mathrm{H} \alpha$ emission in $\mathrm{HH} 223$ corresponds to knots A and B. It is not possible to know whether these $\mathrm{H} \alpha$ knots have any near infrared counterpart, as the $\mathrm{H}_{2}$ image of Palacios \& Eiroa (1999) does not cover the region beyond the east border of $\mathrm{HH} 223$ knot C. We have not found in our $\mathrm{H} \alpha$ image any optical counterpart for the near infrared emission $\mathrm{H} 2-\mathrm{NW}$ as well as for the near infrared knots $\mathrm{K} 1$ and $\mathrm{K} 2$, found a few arcsec to the east of the VLA2 radio source (see Fig. 1). The lack of detection of optical counterparts of these near infrared emissions is most probably due to the higher visual extinction. However, it is interesting to point out that we have barely detected diffuse $\mathrm{H} \alpha$ nebulosities farther away $(\sim 1,5)$ to the west of the VLA radio sources. We have labeled these features HH 223-NW1 and HH 223-NW2 (see Table 1 and Fig. 1). These faint $\mathrm{H} \alpha$ emissions features are found nearly aligned with the $\mathrm{H}_{2}$ condensations $\mathrm{K} 1, \mathrm{~K} 2$ and $\mathrm{H} 2-\mathrm{NW}$ of Palacios \& Eiroa (1999).

As it was first pointed out by Vrba et al. (1986), HH 223 has a nearly east-west orientation. Let us define the HH 223 outflow axis passing through knots A-E-F3 and through the nebulosity N2 (Fig. 3). This direction defines the overall orientation of $\mathrm{HH} 223$, taking into account the fainter $\mathrm{H} \alpha$ nebular emission in which the knotty structure is found engulfed. The emission axis defined in this way has a $\mathrm{PA} \simeq 96^{\circ}$. This orientation is very close to the magnetic field direction measured by Vrba et al. (1986) $\left(\mathrm{PA}=90^{\circ} \pm 8^{\circ}\right)$ and to the axis of the east-west CO outflow $\left(\mathrm{PA}=110^{\circ}\right)$. But in addition, our deeper $\mathrm{H} \alpha$ image allows to distinguish some "wiggling" in the HH 223 knot pattern: knots C-D-E-F1 are oriented following a direction with a PA of $79^{\circ}$. Nearly the same orientation is obtained for the knots A-B direction $\left(\mathrm{PA}=80^{\circ}\right)$. Thus, despite the $\mathrm{H} \alpha$ low-emission presents a nearly east-west orientation, the knotty struture embedded inside the nebula is organized in two nearly parallel chains of knots, being these chains oriented in a slightly different direction within the low-emission nebula.

\subsection{2. [SII] emission}

HH 223 also shows in the [SII] lines the knotty, wiggling structure found in $\mathrm{H} \alpha$ (Fig. 2): knots from A to D are clearly identified, and [SII] emission is detected at the positions found for knot $\mathrm{E}$ and filament $\mathrm{F}$ in the $\mathrm{H} \alpha$ image. However, it is not possible to resolve in the [SII] image the three condensations (F1, F2 and F3) identified within the F filament in $\mathrm{H} \alpha$. Furthermore, we have not detected [SII] emission coinciding with the positions of the weaker $\mathrm{H} \alpha$ emission nebulosities $\mathrm{N} 1$ and N2.

Interestingly, we can appreciate significant differences by comparing the flux variations from knot to knot in $\mathrm{H} \alpha$ and in [SII]. This fact is more evident for knot $\mathrm{A}$, where the $\mathrm{H} \alpha$ emission is stronger as compared with the rest of the knots, while the strength of the [SII] emission in knots A, B and C is comparable. For the case of shock-excited emission found in stellar jets, information on the degree of excitation of the emitting gas and on the shock velocity can be derived from the [SII]/ $\mathrm{H} \alpha$ line ratio (Raga et al. 1996; Hartigan et al. 1987). Thus, our data suggest variations of the shock-excitation conditions through HH 223.

We show the map (Fig. 4) of the [SII] to $\mathrm{H} \alpha$ flux ratio through $\mathrm{HH} 223$ derived from the subimages mentioned in Sect. 2. We do not intend to compare the numerical values found for the $[\mathrm{SII}] / \mathrm{H} \alpha$ ratio with the line ratio values obtained from modeling the spectra of shock-excited gas. However, our $\mathrm{HH} 223[\mathrm{SII}] / \mathrm{H} \alpha$ map is useful to show clear signs of variation of the excitation conditions through $\mathrm{HH} 223$, as the [SII]/ $\mathrm{H} \alpha$ flux ratio changes up to a factor $\gtrsim 3$ in different $\mathrm{HH} 223$ knots. As a general trend, the [SII]/ $\mathrm{H} \alpha$ flux ratio increases (excitation decreases) from east to west and from north to south through $\mathrm{HH}$ 223. This ratio reaches a minimum around knot $\mathrm{A}$ (the lowest line ratio values are found to the south-east, close to the peak of knot A). The ratio has a steep increase going to the west, reaching the highest values towards the north of the peak of knot B. From the western edge of knot $B$ to knot $C$ the ratio decreases. Beyond knot $\mathrm{C}$, the ratio remains nearly constant, with a slight decrease as we move from east to west and from south to north. We especulate that inhomogeneities, both in the ambient and in the high velocity molecular gas might be contributing to the variations in the excitation conditions through HH 223 The existence of such inhomogeneities is expected since HH 223 is located at an interface, towards the southern edge of a high optical extinction region and, in addition, $\mathrm{HH} 223$ lies coinciding in position with high velocity molecular gas, traced by $\mathrm{CO}$ emission, as can be seen from Fig. 5.

\subsection{Other $\mathrm{H} \alpha$ nebulosities in $L 723$}

We have identified some other nebulosities in the $\mathrm{H} \alpha$ image of the L723 field (see Fig. 1 and Table 1 for identification). The two brighter nebulosities are found to the south-east of $\mathrm{HH} 223$. The eastern one, $\sim 2^{\prime}$ from HH 223 knot A, coincides in position with the object V83 of Vrba et al. (1986), although the line emission appears more extended in our image and because of that, we have renamed it HH 223-SE1. This nebulosity shows an arcshaped morphology, with its apex pointing toward HH 223 and probably has a knotty structure which cannot be well resolved from our image. The other nebulosity, labeled HH 223-SE2, is $\sim 30^{\prime \prime}$ from HH 223-SE1, and appears as a quite bright linear 


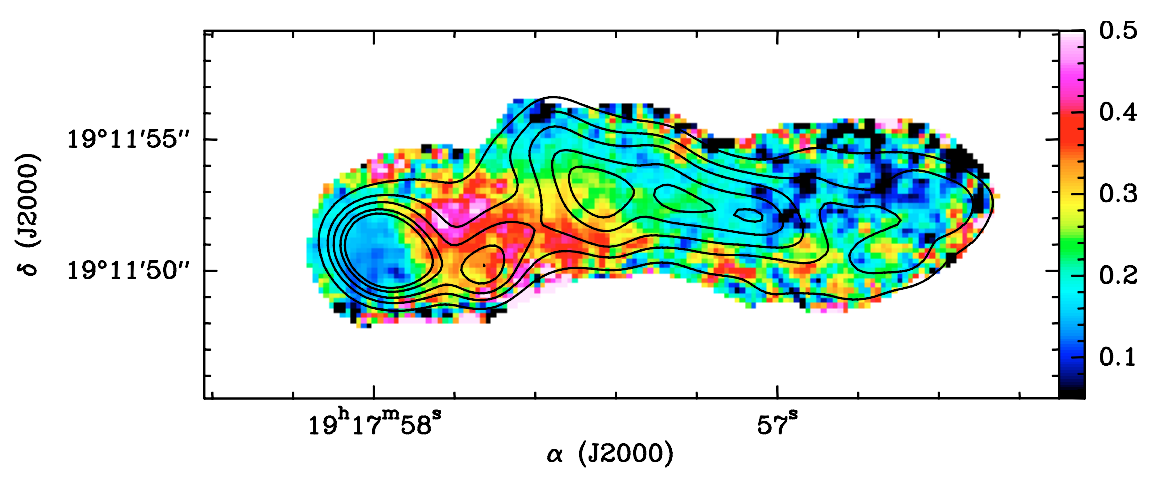

Fig. 4. [SII]/H $\alpha$ flux ratio for $\mathrm{HH} 223$. A contour plot of the $\mathrm{H} \alpha$ line flux has been superposed.

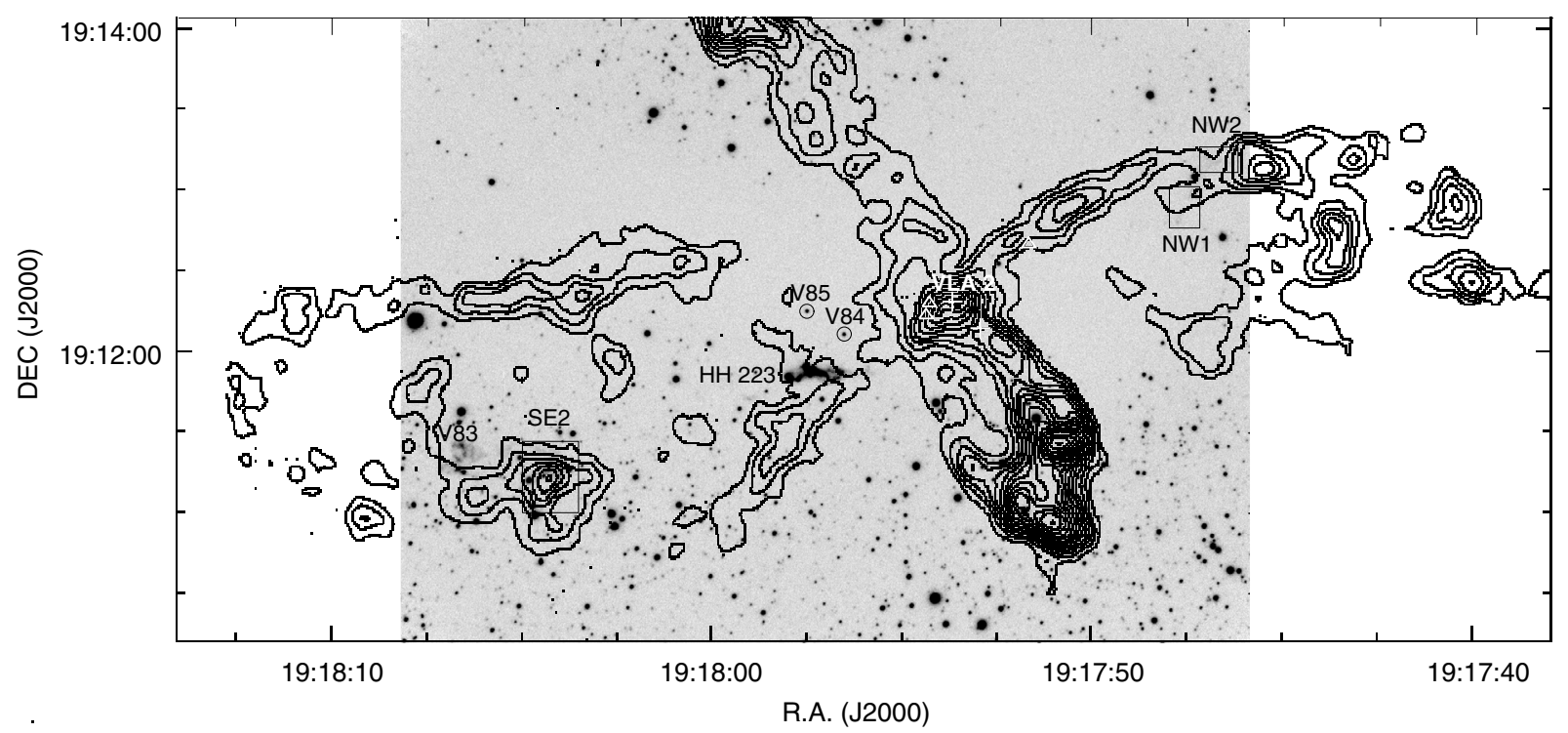

Fig. 5. Image of L723 in $\mathrm{H} \alpha$ with HH 223 in the center of the field, superposed on the CO outflows from Lee et al. (2002). The VLA2 source and the other $\mathrm{H} \alpha$ nebulosities have been labeled. The triangles mark the positions of the $\mathrm{H}_{2}$ emission peaks of Palacios \& Eiroa (1999).

filament $\sim 2^{\prime \prime}$ long emerging from a faint star. Two more nebulosities are barely detected on the $\mathrm{H} \alpha$ image and are found $\sim 3^{\prime}$ to the north-west of HH 223 knot A. These two features are labeled HH 223-NW1 and HH 223-NW2. Interestingly, HH 223-NW2 spatially coincides with a filamentary emission nebula visible on the $K^{\prime}$ image of Hodapp (1994). It is not possible to say from these data whether all these nebulosities have any relationship with the molecular outflows or with the near infrared and optical line emissions found in L723. However, as can be seen in Fig. 5, all of these $\mathrm{H} \alpha$ nebulosities are found nearly aligned along the direction defined by the axis of the east-west bipolar $\mathrm{CO}$ outflow. In contrast, the positions given by Vrba et al. (1986) for the possible $\mathrm{HH}$ objects V84 and V85 coincide in all of our images with the positions of two faint field stars. Furthermore, these two objects are found in the 2MASS All Sky Catalog of Point Sources as two stars with $K$ magnitudes of 10.84 and 9.38, respectively. Thus we discard the HH-like nature of Vrba objects V84 and V85.

\section{Conclusions}

We have obtained deep optical images in the $\mathrm{H} \alpha$ and [SII] lines, and in the continuum nearby $\mathrm{H} \alpha$ of L723. The $\mathrm{H} \alpha$ image allows to resolve the detailed structure of the "linear emission feature" HH 223, reported by Vrba et al. (1986). The emission consists of several knotty condensations engulfed in a fainter nebula. Some wiggling is present in the $\mathrm{HH} 223$ knot pattern. The $\mathrm{H} \alpha$ emission spatially coincides with an $\mathrm{H}_{2}$ elongated emission, although the $\mathrm{H} \alpha$ emission appears more extended. HH 223 is also detected in the [SII] lines and shows the knotty, wiggling morphology found in the $\mathrm{H} \alpha$ line, although the [SII] emission is weaker than the $\mathrm{H} \alpha$ emission. In contrast, no emission was detected in the continuum image at the position of HH 223. Thus, HH 223 appears as a line-emission object whose properties are reminiscent of those found in stellar jets, indicating a shock-excited origin of the emission. Comparison between the [SII] and $\mathrm{H} \alpha$ emission fluxes indicates that there are differences in the excitation conditions through $\mathrm{HH} 223$. As a general trend, the $[\mathrm{SII}] / \mathrm{H} \alpha$ line ratio increases (excitation decreases) from east to west and from north to south of $\mathrm{HH} 223$. The lowest ratio (highest excitation) is found around knot $\mathrm{A}$, while the highest ratio (lowest excitation) is found in a region to the north of the peak of knot B. High resolution spectroscopy of HH 223 would be very helpful to confirm the shock-excited origin of the HH 223 emission as well as to measure the HH 223 radial velocity field. Knowledge on the kinematics of $\mathrm{HH} 223$ would allow to establish whether the HH 223 line emission is supersonic (as in typical stellar jets) or, in contrast, is nearly stationary as would be expected for an illuminated wall of the cavity excavated by the molecular outflow. 
In addition to $\mathrm{HH} 223$, we have detected in the L723 field imaged emission in the $\mathrm{H} \alpha$ line from several other nebulosities. None of these nebulosities appear in the image obtained in the continuum nearby $\mathrm{H} \alpha$. All of these nebulosities are found projected onto the east-west pair of lobes of the quadrupolar L723 CO outflow. The eastern nebulosity coincides with the Vrba object V83, thus confirming the HH-like nature of it. In contrast, we have discarded the Vrba objects V84 and V85 as being $\mathrm{HH}-$ like objects.

Acknowledgements. We acknowledge the Support Astronomer Team of the IAC for obtaining the images. The work of R.E., R.L. and A.R. was supported by the Spanish MEC grant AYA2005-08523-C03-01. ALFOSC is owned by the Instituto de Astrofísica de Andalucía (IAA) and operated at the NOT under agreement between IAA and the NBIfAFG of the Astronomical Observatory of Copenhagen. This publication makes use of data products from the Two Micron All Sky Survey, which is a joint project of the University of Massachusetts and the Infrared Processing and Analysis Center/California Institute of Technology, funded by the National Aeronautics and Space Administration and the National Science Foundation.

\section{References}

Anglada, G., Estalella, R., Rodríguez, L. F., et al. 1991, ApJ, 376, 615 Anglada, G., Rodríguez, L. F., \& Torrelles, J. M. 1996, ApJ, 473, L123 Avery, L. W., Hayashi, S. S., \& White, G. J. 1990, ApJ, 357, 524 Cabrit, S., \& André, P. 1991, ApJL, 379, 25

Girart, J. M., Estalella, R., Anglada, G., et al. 1997, ApJ, 489, 734

Goldsmith, P. F., Snell, R. L., Hemeon-Heyer, M., \& Langer, W. D. 1984, ApJ, 286, 599

Hartigan, P., Raymond, J., \& Hartmann, L. 1987, ApJ, 31d6, 323

Hirano, H., Hayashi, S. S., Umemoto, T., \& Ukita, N. 1998, ApJ, 504, 334 Hodapp, K. W. 1994, ApJS, 94, 615

Lee, C. F., Mundy, L. G., Stone, J. M., \& Ostriker, E. C. 2002, ApJ, 576, 294 Palacios, P., \& Eiroa, C. 1999, A\&A, 346, 233

Raga, A. C., Böhm, K.-H., \& Cantó, J. 1996, Rev. Mex. Astrofis. Astron., 32, 161

Reipurth, B. 1994, A general catalogue of Herbig-Haro objects, Electronic version 1994-1

Reipurth, B., Chini, R., Krügel, E., Kreisa, E., \& Sievers, A. 1993, A\&A, 273, 221

Vrba, F. J., Luginbuhl, C. B., Strom, S. E., Strom, K. M., \& Heyer, M. H. 1986, AJ, 92, 633 